\title{
A simulation model for unsignalized pedestrian crosswalks based on evidence from field observation
}

\author{
Claudio Feliciani ${ }^{\mathrm{a}, *}$, Luca Crociani ${ }^{\mathrm{b}}$ Andrea Gorrini ${ }^{\mathrm{b}}$ Giuseppe Vizzari ${ }^{\mathrm{b}}$ Stefania Bandini ${ }^{\mathrm{b}}$ and \\ Katsuhiro Nishinari ${ }^{c, d}$ \\ ${ }^{a}$ Department of Advanced Interdisciplinary Studies, Graduate School of Engineering, The University of Tokyo, \\ Tokyo 153-8904, Japan \\ b Complex Systems and Artificial Intelligence Research Centre, University of Milano-Bicocca, Viale Sarca 336/14, \\ 20126, Milan, Italy \\ ${ }^{\mathrm{c}}$ Research Center for Advanced Science and Technology, The University of Tokyo, Tokyo 153-8904, Japan \\ ${ }^{\mathrm{d}}$ Department of Aeronautics and Astronautics, Graduate School of Engineering, The University of Tokyo, Tokyo \\ 113-8656, Japan
}

\begin{abstract}
This paper presents a model to simulate unsignalized pedestrian crosswalks. Principal scope of the model is to develop a tool to be used by decision-makers to evaluate the necessity of introducing a new crosswalk and/or switching to a traffic light and estimate the potential benefits of such a measure in term of Level of Service. The model is based on empirical evidence gained during an observation of an unsignalized crosswalk in Milan. Pedestrian motion is simulated using a simple Cellular Automata model in which only static floor field is implemented. Vehicles use a continuous car following model inspired on Gipps equations in which driver's reaction time is considered. Pedestrian's decision-making process on crossing attempt and model parameters are directly obtained from the analysis of pedestrian-vehicle interactions observed in reality. The model developed employs small time steps, thus allowing the consideratinn $n$ f different pedestrian speeds (intrinsically allowing to consider elderly) and smoothly reproducing car-pedestrian interaction order to validate the model, delays (or waiting times) measured for both pedestrians and drivers were compared with simulau values. Results show a good agreement between empirically obtained time delay and values computed in the simulation.
\end{abstract}

Keywordc. Keywords: unsignalized crosswalk, vehicular traffic, pedestrian traffic, traffic simulation, pedestrian-vehicle interact

\section{Introduction}

Traffic accidents have occurred since the introduction of the first engine powered vehicles with initial reported cases dating back at the end of the 20th century [41]. Before the problem became widespread legislators had already started improving the safety of road users by clearly separating pedestrian and vehicular flows. Traffic lights were first installed in the United

\footnotetext{
${ }^{*}$ Corresponding author. E-mail: feliciani@jamology.rcast.utokyo.ac.jp.
}

Kingdom in 1868 and in 1890 it was suggested to create under/overpasses to physically separate the road surface used by pedestrians and vehicles [41].

More than one century later road safety has greatly improved, with the number of road fatalities reported in the United Kingdom passing from a maximum of 7'952 in 1965 to 3'172 in 2006, this although the number of licensed vehicles has almost tripled [7]. In Italy a similar trend has been observed with a peak of 11'078 road casualties registered in 1972 when the country fleet size was about 15 million vehicles. The number of fatalities has rapidly decreased reaching 4'090 
in 2010 (with the number of registered vehicles more than tripled) [22]. However, although in Italy the number of casualties has kept decreasing for a number of years, after 2013 this figure has slightly increased making it questionable whether the objective of 2'057 road traffic victims set for 2020 could be effectively reached [21].

When road safety is considered on a global scale the situation appears different, with emerging economies seeing an increase in road traffic causalities, both in absolute and relative terms. For example, in India traffic fatalities per 100'000 population were about 3 in the 70's but passed to 8 at the end of the 21 st century [23]. The situation appears particularly dramatic in south Asia where the number of death in traffic accidents is predicted to more than double during the 2010-2020 period. Globally, traffic injuries were the 11. leading cause of death with the number of fatalities only slightly below the ones caused by malaria [23]. Among all road users, pedestrians are some of the most vulnerable, with the percentage of pedestrians fatalities lying at $25 \%$ of the overall traffic victims in the United Kingdom in 2014 [8] and at 17\% in Italy in 2015 [21]. In developing countries pedestrians appear to be also vulnerable road users, in particular in India where they represent the majority of road users killed in accidents with a proportion over $40 \%$. The percentage grows when large cities are considered and in Mumbai $78 \%$ of the traffic victims are pedestrians (53\% for New Dehli). Worldwide, crashes between vehicles and pedestrians account for more than a third of all road-traffic deaths and injuries [23].

Although in modern countries the number of pedestrians casualties has rapidly decreased in line with a general improvement of road safety and compliance, this figure has been almost unchanged in the recent years. In the United Kingdom the number of pedestrians killed in road accidents has been fairly constant since 2010 [8] and in Italy an increase of $4.2 \%$ has been recorded for the period 2014-2015 [21]. In the U.S. the number of pedestrian fatalities has not shown any significant trend since 2004 [46], with pedestrians still representing $14 \%$ of all fatal crashes in 2013 .

While the increasing number of pedestrian fatalities in developing economies can be related to the overall increase of motorized traffic and non-compliance issues, the recent increase of pedestrian's crashes in developed countries appears to be more difficult to explain. Some studies suggest that one of the possible causes for this increase is related with elderly pedestrians $[3,8]$, which has often been identified as being one of the most vul- nerable group of pedestrians $[1,38]$. Although most of the fatalities recorded for pedestrians are relative to the 15-29 age group, results drastically change when the proportion of each age group inside the population is taken into account $[1,23]$. Clearly, the reason for the high relevance of fatalities in elderly pedestrians is related to different factors associated with physiological limitations given by their age. In particular, factors limiting their capability to interact with road users are given by: (i) their limited locomotion capabilities (e.g., reduced muscle strength and coordination abilities, posture differences and low walking speed); (ii) the reduced reliability of perceptive sensors and cognitive functions (e.g., ability to distinguish colors and evaluate lighting conditions, inefficient suppression of background noise, poor attention and reaction time, disorientation and slower decision-making) [14]. Concluding, the importance in improving safety for elderly pedestrians is clearly reported in the "World Report on Road Traffic Injury Prevention", which highlights pedestrian safety as the main safety concern for the elderly road users.

Some of the most critical traffic components in which pedestrians and drivers interact are represented by crosswalks, with unsignalized ones creating an higher risk for pedestrians compared with the one signalized using traffic lights. For example, in the case of Israel, elderly pedestrians crossing the road in locations within a metropolitan/urban area were found as the most common type of road accident leading to death [38].

However, research on pedestrian behavior on crosswalks has been limited by a variety of factors: difficulty in obtaining empirical data through on-field observations, safety and ethical concerns related with experiments involving individuals and the limited scope of the simulation models developed in the past. More recently virtual reality is offering the possibility to evaluate driver and pedestrian reaction in an environment very close to reality without the risks which would exist in a real scenario [29,30,39]. But still, although a variety of simulation software can deal with traffic from both a macroscopic and microscopic point of view, only little attention has been put in developing simulation models to reproduce crossing behavior on the basis of empirical data collected in real situations. Such a simulation tool is required if pedestrian safety and comfort has to be improved and a development in this direction would also lead to an increased accuracy on the macroscopic scale. In addition, a reliable simulation model would allow policy-makers to take deci- 
sions based on a more rigorous base thus contributing to the creation of more safe and comfortable areas for pedestrians and road users.

This paper is organized as follows: chapter 2 provides a literature survey of research on unsignalized crosswalks covering both experimental studies and modeling attempts. Chapter 3 presents in detail the simulation model for this study and in chapter 4 the main results are presented. Finally chapter 5 will provide a conclusive discussion.

\section{Literature survey}

As the numbers above testify, it is rather easy to find statistical data concerning the relevance of a certain type of accident occurring to pedestrians and most of the countries make those data openly available. However, it is more difficult to find reliable studies describing the behavior of pedestrians in crosswalks and this may be one of the reasons why simulation models developed so far could not be validated using empirical data. Hereafter, we will attempt to summarize some of the most relevant studies considering the interaction between pedestrians and vehicles at unsignalized crosswalks (when relevant, research on signalized crosswalks will be also considered). We will summarize empirical studies at first and later focus on modeling attempts. Finally, we will summarize the empirical aspects on which our model is based.

\subsection{Empirical studies}

Most of the experimental studies on pedestrian crosswalks focused on measuring walking speed of pedestrians and investigating possible differences with behavior observed in pedestrians in common situations. Pedestrian speed at crosswalks was found roughly following a normal distribution [12,20,24,32, 37], which should not come as a surprise since pedestrian walking speed in general has been long known for being normally distributed $[10,19,49]$. Reported crossing speed change depending on the type of crosswalk considered (signalized or unsignalized) and the physical characteristic of the pedestrian walking on it, with gender and age being statistically relevant (young and males are significantly faster than elderly and women). On the other side, ethnicity of the pedestrian, grouping pattern and lighting condition of the crosswalk were not found to significantly impact the crossing speed $[12,32]$. In addition, in the case of signalized cross- walks pedestrians were shown having significantly higher crossing speeds when attempting to cross in the "don't walk" time [37]. Speed-density relation for the situation of signalized crosswalk showed a linear relationship similar to the one observed in general pedestrian facilities $[37,49]$.

Other researchers have set their attention on drivers' behavior and speed profiles observed while approaching a (unsignalized) zebra crossing. It was found that drivers do not follow the law concerning speed behavior and that instead, maintaining high speeds or even accelerating was used to signalize to pedestrians that they do not intend to give the way. The region lying between 50 to $40 \mathrm{~m}$ before the zebra crossing was found critical for influencing drivers' behavior [47]. Pedestrians' capability to estimate approaching car's speed vary by weather conditions and vehicle speed. If the speed of the oncoming vehicle exceed an upper bound, pedestrians are more likely to underestimate vehicle speed, thus increasing the risk of trying dangerous crossing attempts [44].

Waiting times for pedestrians attempting to cross zebra crossings are typical of the location considered, but in general short waiting times are much more frequent than long intervals, with the distribution rapidly decreasing the as the waiting time grows bigger [20]. In a particular study [16], waiting time has been analyzed based on a number of factors which can potentially have an influence in reducing it, consequently trying risky crossing attempts. Results show that female pedestrians, those having children, those who own and drive a car, elderly pedestrians and people having assisted to traffic accidents are more likely to accept longer waiting time at the curbside. Among factors influencing pedestrians toward more risky behavior were found commuting to work, frequent use of the given crosswalk and crossing in group behavior [16]. Concerning crossing attempts, it has been shown that slightly less than half of pedestrians $(41.67 \%)$ try to cross at the first attempt and that expected waiting time seems to influence the number of attempts required to cross the street [16]. It has been shown that the perception of ease in regard with the crossing task influences the degree of hazard taken by pedestrians when attempting to cross a road. Those thinking it would be easy to cross are more likely to take risks [9].

Several researchers identified the acceptance gap as one of the main parameters unconsciously used by pedestrians in judging if a crossing attempt can be considered safe or not. The accepted time gap can be defined as the relation between the distance (along the 
driving direction) and the average speed of oncoming vehicles when pedestrians decided to cross [14]. Although data on this aspect are limited, researchers seem to agree that accepted gaps are in the order of 4-6 seconds [14,24,50]. Distributions for accepted and refused gaps are different, but both tend to flatten when accepted gaps get longer and refused gaps shorter. Interestingly, the distributions for accepted gaps by pedestrians waiting and not waiting only differ to a small extent [24]. Finally, time gap (or vehicle headway to use another expression) was found having a positive coefficient toward waiting time, meaning that waiting time seems to decrease for short time gaps.

Other studies considered aspects generally related with city planning and infrastructure selection. A comparative study considered different types of pedestrian crossings (signalized, unsignalized, marked, nonstriped, midblock crosswalks,...) and analyzed pedestrians preference and compliance observed for each type. Unsignalized midblock crosswalks were among the preferred by pedestrians and high crossing compliance was also observed. However, location of the crosswalk relative to origin and destination was found being the most influential decision factor. Less incisive but still remarkable were the presence of traffic control and vegetation or concrete barriers [42]. The relationship between pedestrian accidents and infrastructure selection has been investigated by considering different types of intersections: three-legged, four-legged, roundabouts and road segments. A function predicting accident's frequency in relationship with pedestrian and traffic flow was derived for each intersection [13].

To conclude this part, a study [17] considered the effect on mobile phone use on the pedestrian crossing behavior at signalized and unsignalized crosswalks. Both males and females surveyed in the study were found crossing at lower speed and taking less attention to surrounding traffic when using mobile phones compared to pedestrians not making use of it.

\subsection{Previous modeling attempts}

Vehicular traffic has been studied for several decades and both mathematical formulations and more sophisticated models are available to perform simulation on traffic flow (an extensive summary including several modeling approaches is given in [34]). Modern software are commercially available to study road networks extending from small neighborhood to large cities. In addition, multiple lanes models have also been developed to simulate lane change and overtaking maneuvers [35].

Pedestrian models have also reached a considerable degree of accuracy. At the present, both continuous and discrete models are successfully employed in a variety of applications, ranging from scientific research to crowd management (a comprehensive review of pedestrian modeling techniques is given in [36] and [48]). Specific models designed to deal with elderly pedestrians have been developed by considering different walking velocities for each agent used in simulation [4].

Although both car's and pedestrian's model have been constantly developed separately for decades, attempts to combine both road users are more rare. One of the first modeling attempt to describe unsignalized pedestrian crossing is the mathematical model developed by Griffiths [15]. This model, however, allows only qualitative considerations of purely mathematical nature and it is difficult to adapt to real-life scenarios. Helbing et al. [18] also developed a mathematical model which has been able to grasp delay caused by the intersecting flows of car and pedestrians. However, although this model allows a rigorous analytical treatment of the problem, its foundation is based on many-particle streams, which does not present some of the heterogeneous aspect found in traffic flow, especially if elderly are considered.

Lawniczak et al. [27,28] proposed a simple Cellular Automata model to simulate the hypothetical case of creatures crossing a highway. In their model, priority is not set on accurately capturing behaviors found in nature, but rather to create a cognitive process in which each creature try to cross the highway and following creatures learn from mistakes observed by their predecessors. Knowledge is transferred from one creature to the following one by judging the behavior which resulted in successful and failed crossing attempts.

Daganzo et al. [6] have considered a similar case in which pedestrians are allowed to cross at any point of a given road, the so-called "pedestrianized streets" (to use their own words). Analytical formulas has been derived to assess the impact of pedestrian's flow on vehicular traffic. The researcher qualitatively concluded that the street's capacity is inversely proportional to the square root of the pedestrian flux for low pedestrian densities. Yang et al. [50] developed a Cellular Automata model to simulate pedestrian behavior at signalized crosswalks. Although their model is designed for pedestrians crossing in the presence of traffic light, the main goal of their study is to implement 
two types of behavior observed during red light period: law-obeying pedestrians (waiting until the traffic light turns green) and opportunistic ones (crossing while facing red light). To account for interaction between pedestrians and vehicles in the crossing attempts with red light a distribution for the accepted time gap is used. From this point of view, the model by Yang et al. intrinsically contains behavioral mechanisms proper of unsignalized crosswalks.

More recently, Zeng et al. [52] developed a social force model to simulate pedestrians' behavior at signalized crosswalks. Similarly to the model by Yang et al., also in the approach by Zeng et al. interactions with drivers are considered to account for the effect of turning vehicles which (compliantly) invade the zebra crossing during green light periods. The simulation by Zeng et al. considers a number of phenomena (individual interactions, group behavior, vehicle interaction, speed changes and deviations from zebra stripes) by modeling them using repulsive or attractive forces of different magnitude. Their model predicts very well pedestrians' trajectories observed in reality by even reproducing pedestrians walking outside the boundary, which are usually not considered in typical social force models. However, interaction with incoming vehicles was only considered in terms of deviation from the normal crossing trajectory and compliance has not been an issue in their study.

Finally, Crociani et al. [5,43] developed a model with the specific intention to consider both signalized and unsignalized crosswalks. In their model cars move in a continuous space environment, while pedestrians' motion is simulated using a standard floor field Cellular Automata model. Interactions at the crosswalks are modeled in an idealistic way: drivers will stop every time a waiting person is spotted on the curbside and pedestrians are capable of precisely estimate car breaking distance based on its speed. One of the limitations of their model is the constant time step (which has to be chosen equal to the drivers' reaction time) which does not allow a smooth variation of pedestrians' walking speed. Also, empirical data have not been used to validate their model, constraining the suitability in evaluating real-life situations.

\subsection{Short summary of field survey in Milan and main results}

Creating a universal model able to reproduce pedestrian behavior observed in different countries and accounting for different type of vehicles would be an extremely difficult and not necessarily indispensable task. On the other side, any new knowledge gained while analyzing a specific traffic scenario will contribute to a better understanding of human behavior in streets, eventually allowing to create increasingly complex and generalized models. It is therefore important to define strength and limitations of the model to be developed and clearly illustrate the context on which it can be applied (scope of application). In the case of this study, rules and parameters implemented into the model have been obtained based on an observation performed in Milan on a unsignalized crosswalk. Main results of the field survey and empirical knowledge used to develop the simulation are presented in this section (a complete analysis of the results with detailed information is given in [14]).

An unsignalized crosswalk in a residential area of Milan (Italy) has been chosen for a roughly hourlong observation. The specific location has been selected based on a number of criteria: the high number of accidents with pedestrians reported, a proportionally large elderly population and strategical factors (proximity with public facilities, possibility to obtain video recordings from a tall building,...). A schematic representation of the crosswalk studied is given in Fig. 2. A camera has been used to record a video (for which a snapshot is given in Fig. 1) which has been later analyzed by means of tracking software and manual analysis. During the 1:15-long observation 1379 vehicles and 585 pedestrians have passed through the target crosswalk. However, after excluding biased vehicle-pedestrian interactions (parked car obstructing the view, turning vehicles, bicycles interfering pedestrians' movements, bikes overtaking cars,...), only a dataset with crossing actions involving 50 pedestrians and 79 vehicles (mostly cars) has been used to study in detail the relationship between different factors in determining pedestrian's decision on crossing or not.

Vehicular traffic throughout the whole observation was evenly distributed in both directions with a large proportion of cars $(67 \%)$, followed by bikes $(13 \%)$, vans $(8 \%)$, cycles $(6 \%)$ and heavy vehicles $(6 \%)$. Measured traffic volume accounted to 1139 vehicles per hour for both directions. Speed limit in the considered section is of $50 \mathrm{~km} / \mathrm{h}$, although vehicles have been rarely observed exceeding a speed of 35-40 km/h (with a partial exception for bikes) mostly because of the consecutive presence of several crosswalks before and after the one analyzed. Among pedestrians, many of them have been observed crossing from South to North (relative to the orientation of Fig. 2), with 


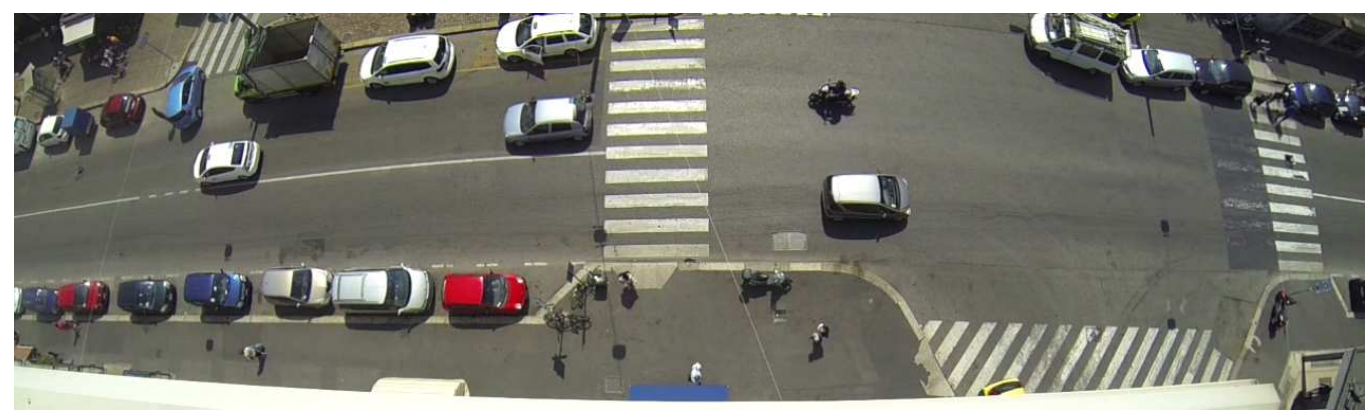

Fig. 1. Snapshot from the video relative to the observation in Via Padova.

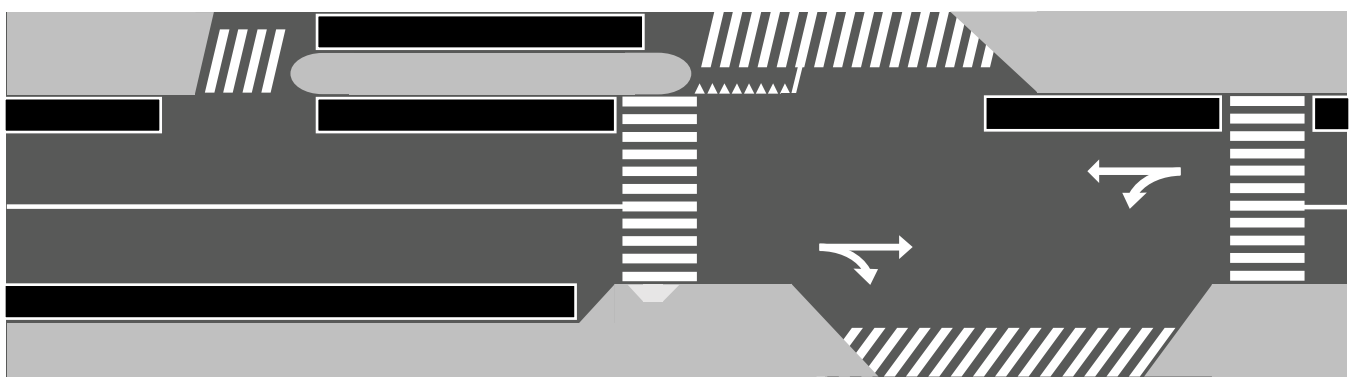

Fig. 2. Schematic diagram of the investigated crosswalk [14].

the largest part being composed by adults (71\%) followed by elderly (24\%). Most of the pedestrians were walking alone $(65 \%)$, with groups dominated by dyads $(26 \%)$ and triples $(8 \%)$. Pedestrian flow on the crosswalk stood at 8.01 pedestrians per minute. However, if groups are considered as a "single entity" because of their cohesion and synchronized motion, the flow for crossing sets (or blocks) reduces to 5.52 per minute.

The so-called "Level Of Service" (LOS) has been used to grade the quality of the crosswalk facility. The idea behind the LOS (for which details are given in [31]) is to compute the time lost due to congestion/delay formed in a given infrastructure (highway, intersection,...) and rate its quality/safety on a graded scale. In the case of crosswalks, both the time lost by pedestrians and drivers need to be considered. For drivers, braking, queuing, waiting and accelerating are actions considered to contribute to the time loss. For pedestrians, the time spent while waiting and during start-up at the curbside is used.

In the case of the crosswalk analyzed, delay (or time loss) for both road users was found as $3.20 \pm 2.73 \mathrm{~s}$ per vehicle and $1.29 \mathrm{~s} \pm 0.21$ per pedestrian. In the grading used in the LOS, this corresponds to a A-level (see Table 1), meaning that nearly all drivers found freedom of operation and that no pedestrians crossed irregularly (therefore taking very few risks).
Analysis of the velocities from selected tracked pedestrians revealed that 3 phases are characteristic in a crossing attempt. During the approaching phase pedestrians move toward the crosswalk; here velocities are equivalent to the ones observed during common situations with only density and social structure influencing it. Around $3 \mathrm{~m}$ before reaching the curbside (this figure slightly varies among individuals), pedestrians slow down and start assessing the viability of their crossing attempt. If it is considered safe to cross, pedestrians will accelerate (eventually reaching a walking speed slightly higher than their approaching speed) and walk on the opposite side of the crosswalk. Walking speeds during the 3 phases are different between adults and elderlies (values are reported in Table 2), although in general elderly pedestrians tend to consistently walk about $0.25 \mathrm{~m} / \mathrm{s}$ slower.

Table 2

Velocity during different crossing phases (relative for the selected pedestrian-vehicle interaction dataset) [14]

\begin{tabular}{lll}
\hline Crossing Phases & Adult pedestrians & Elderly pedestrians \\
\hline Approaching speed & $1.28 \pm 0.18 \mathrm{~m} / \mathrm{s}$ & $1.03 \pm 0.18 \mathrm{~m} / \mathrm{s}$ \\
Appraising speed & $0.94 \pm 0.21 \mathrm{~m} / \mathrm{s}$ & $0.69 \pm 0.23 \mathrm{~m} / \mathrm{s}$ \\
Crossing speed & $1.35 \pm 0.18 \mathrm{~m} / \mathrm{s}$ & $1.09 \pm 0.17 \mathrm{~m} / \mathrm{s}$ \\
\hline
\end{tabular}


Table 1

The Level of Service criteria for two-way stop-controlled unsignal-

ized intersections [31]

\begin{tabular}{|c|c|c|c|}
\hline LOS & Description & Vehicular Delay [s/veh] & Pedestrian Delay [s/ped] \\
\hline A & $\begin{array}{l}\text { - Nearly all drivers and freedom of operation } \\
\text { - Very small delay, none crossing irregularly }\end{array}$ & $<5$ & $<10$ \\
\hline B & $\begin{array}{l}\text { - Occasionally there is more than one vehicle in queue } \\
\text { - Small delay, almost no one cross irregularity }\end{array}$ & $5-10$ & $10-15$ \\
\hline $\mathrm{C}$ & $\begin{array}{l}\text { - Many times there is more than one vehicle in queue } \\
\text { - Small delay, very few pedestrian crossing irregularity }\end{array}$ & $10-20$ & $15-25$ \\
\hline $\mathrm{D}$ & $\begin{array}{l}\text { - Often there is more than one vehicle in queue } \\
\text { - Big delay, someone start crossing irregularity }\end{array}$ & $20-30$ & $25-35$ \\
\hline $\mathrm{E}$ & $\begin{array}{l}\text { - Drivers and the delays approaching intolerable levels } \\
\text { - Very big delay, many pedestrians crossing irregularity }\end{array}$ & $30-45$ & $35-50$ \\
\hline $\mathrm{F}$ & $\begin{array}{l}\text { - Forced flow due external operational constraints } \\
\text { - Pedestrian cross irregularly, engaging risk-taking behaviors }\end{array}$ & $>45$ & $>50$ \\
\hline
\end{tabular}

Finally, the decision-making process for pedestrians attempting to cross the road has been analyzed by using the time gap previously discussed. Time gaps are obtained by measuring the distance and speed of each vehicle at the time pedestrians decide to cross the road (this moment is represented by the transition between appraising and crossing phase). Results for the 50 pedestrians considered in the dataset are given in Table 3.

Table 3

Measured car's quantities at the beginning of the crossing phase [14]

\begin{tabular}{lll}
\hline Quantity & Adult pedestrians & Elderly pedestrians \\
\hline Car distance & $16.21 \pm 8.09 \mathrm{~m}$ & $17.61 \pm 9.50 \mathrm{~m}$ \\
Car speed & $16.54 \pm 6.48 \mathrm{~km} / \mathrm{h}$ & $15.15 \pm 7.68 \mathrm{~km} / \mathrm{h}$ \\
\hline
\end{tabular}

Although deviations are very large, distance from cars accepted by elderly when moving out from the sidewalk are larger compared to adult pedestrians. On the other hand speed of cars appear to be smaller. Based on those two data, it is possible to compute the accepted time gap by simply dividing distance and speed. Results are presented in Table 4 with distinction being made for near and far lane.

Table 4

Accepted time gaps for different types of pedestrians and each lane [14]

\begin{tabular}{lll}
\hline Time gap & Adult pedestrians & Elderly pedestrians \\
\hline Near lane & $3.84 \pm 2.87 \mathrm{~s}$ & $4.43 \pm 1.72 \mathrm{~s}$ \\
Far lane & $4.22 \pm 1.96 \mathrm{~s}$ & $4.59 \pm 1.92 \mathrm{~s}$ \\
\hline
\end{tabular}

Again, elderly appear to be more caution when deciding to cross the road, although deviations are very large making differences statistically insignificant. Differences are smaller when the far lane is considered.

The model that we will introduce in the next chapter is based on the qualitative aspects apprehended while visioning the video recording and empirical evidence gained. In addition, numerical values obtained in the above analysis has been used to calibrate the model.

\section{Simulation model}

As shortly discussed above, Crociani et al. already developed a model to simulate interactions between vehicles and pedestrians at unsignalized crosswalks. However, their model had several shortcomings and it was not suitable to use the empirical results previously introduced for calibration. The model presented here represents a substantial improvement from the initial modeling attempt and it allows to perform calibrations based on empirical data gained from field observations.

In this chapter, the simulation model will be discussed in detail, including geometrical setup for the road (and crosswalk), algorithms to sequentially move pedestrians and cars and the rules governing interactions between both road users.

\subsection{General structure}

The simulation code has been written using Repast Simphony (version 2.4), which has several libraries dedicated to multi-agent simulations and particularly suits to the case of traffic dynamics. The simulated 
geometry corresponds to the scenario of the observation, whose dimensions are given in Fig. 3. The portion of road considered has a single crosswalk in the center where pedestrians and cars interact. The crosswalk section contains a "virtual" midblock which allows pedestrians to stop in the middle of the street without colliding with incoming cars (details for this approach will be presented below).

Cars move on each of the two lanes in opposite directions. Periodic boundary conditions are used to allow a continuous flow of cars: once a car leaves from one side of the road it is (re)created in the next computational step on the opposite side. Pedestrians are randomly generated from one of the cardinal points indicated in Fig. 3. Origin and destination are randomly chosen by ensuring that the chosen combination will force the given pedestrian to pass through the crosswalk. After reaching the destination each pedestrian is removed from the scenario.

Car and pedestrian environment is modeled using two different approaches, allowing to use the best approach for each type of agent (a schematic representation is given in Fig. 4). Cars are modeled in a continuous space, in which the position along the lane can be computed in an accurate way limited only by the machine precision (car position will consequently be given as a decimal number). This approach is necessary since cars move with a large range of velocities and a discrete approach would not allow to accurately compute positions at low and high velocities. Pedestrian sub-environment is made using a grid composed of square cells of fixed side length (size is chosen based on typical literature values). Each pedestrian can occupy one cell at a time, thus ensuring that maximum density for human crowd is not crossed even when all cells are occupied. Pedestrians can only move in the portion of the grid which is not occupied by the road, with the exception of the crosswalk where both pedestrians and cars are allowed to pass. The advantage of this type of environment is to allow a subdivision between the space occupied by each agent, thus making it possible to simulate each road users in an independent way and considering only interactions as a connective point between both agents.

\subsection{Pedestrian model}

Pedestrian motion inside the computational grid is based on the floor field model, which will be presented here shortly (for a detailed review on different floor field approaches see $[36,40,48])$. By using the Moore neighborhood and assuming that a pedestrian is located in the black cell indicated in Fig. 5, there are 8 possible cells in which the pedestrian may move in a single step: the 4 cells lying at each side and the 4 cells located at the corners.

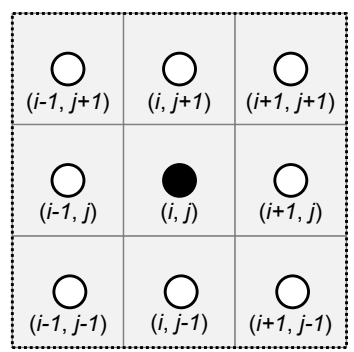

Fig. 5. Pedestrian mesh using Moore neighborhood.

In the floor field model the selection for a cell to move in is based on a probability assigned to each cell. In order to "guide" each pedestrian to a preferred destination, the closest the cell will be to that destination the lowest will be the value assigned to the cell. For the case of the scenario considered here an illustrative example of the static floor field (i.e. the field guiding the pedestrian) is provided in Fig. 6 .

The example provided here represents the case of a pedestrian aiming to the SW-exit (see also Fig. 3 for reference). Clearly, the smallest values of the static floor field are found at the left-lower corner. As a consequence, the pedestrian will be directed there, with no regard on which corner is generated. By looking again at Fig. 3 it can be noticed that the field strength is symmetrical in both sides of the upper section of the street. This means that if a pedestrian is generated at the upper sidewalk (no matter if left of right), he will have to cross the road to reach his destination.

In the most simple implementation of the floor field model each pedestrian determine his next cell during each time step only based on the static floor field discussed before. Walking speed is simply considered by adjusting the size of time step and cell size. Typically $40 \mathrm{~cm}$ cells and time steps in the order of $0.3 \mathrm{~s}$ are used, with a resulting free walking speed of about $1.3 \mathrm{~m} / \mathrm{s}$. However, in our model we wish to consider different walking speeds and a small time step is also required to accurately represent car behavior. It is therefore important to consider a system allowing to set pedestrian individual walking speeds. An idea would be to have pedestrians moving to the next cell with some probability computed to match with the actual walking 


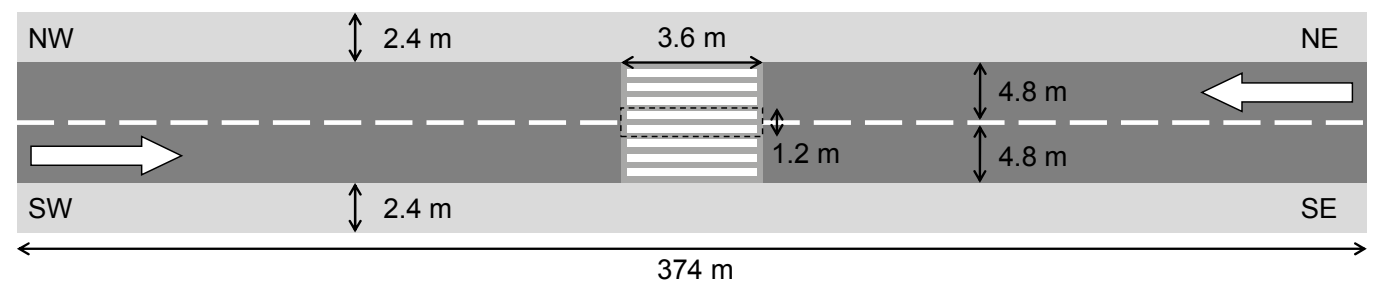

Fig. 3. Schematic representation with dimensions of the simulated model (dotted area is the "virtual" midblock).
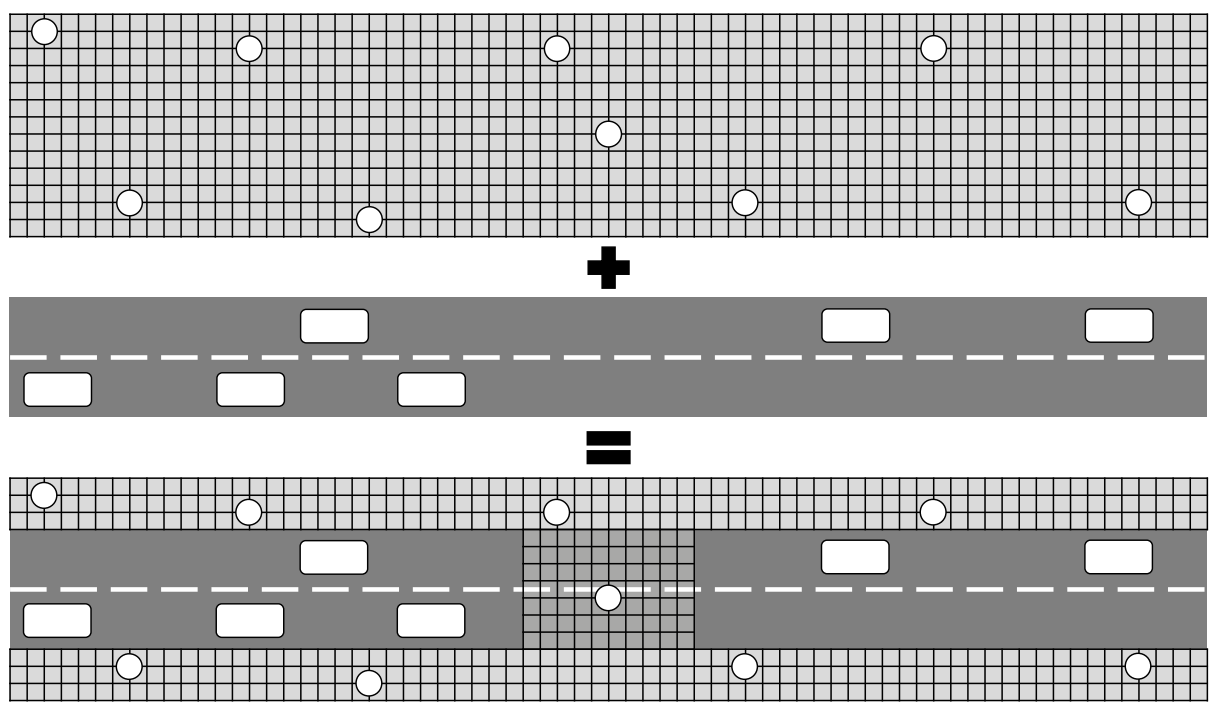

Fig. 4. Representation of the global environment created by superposition of the vehicular and pedestrian sub-environments.

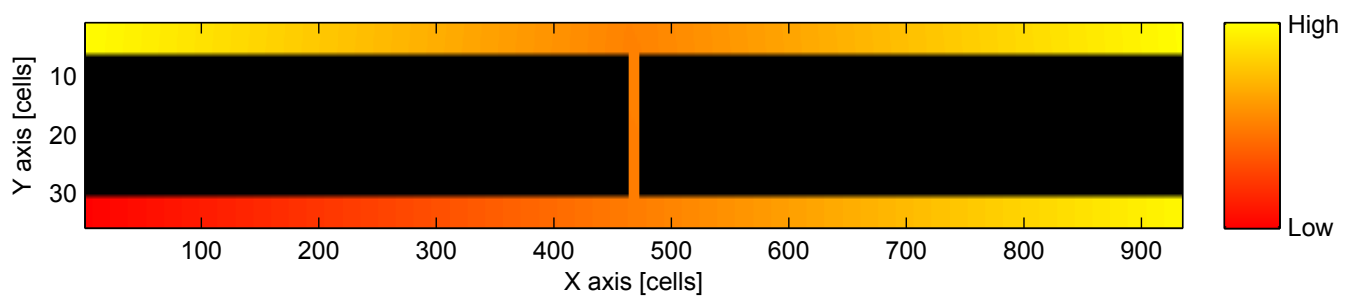

Fig. 6. Static floor field for the scenario considered in simulations (pedestrian destination is South-West).

speed. This "update probability" $\mu$ can be computed as:

$$
\mu=v_{\text {ped }} \frac{t_{\text {step }}}{s_{\text {cell }}}
$$

where $v_{p e d}$ is the (desired) pedestrian speed, $t_{\text {step }}$ is the time step and $s_{\text {cell }}$ is the side length of the grid's cell. For each iteration a random number $r=[0,1]$ is generated and the pedestrian is moved to a neighbor cell if $r<\mu$. The method is simple and efficient, but real moving speed tends to have large oscillations as shown in Fig. 7.

This happens because of the probabilistic nature of the method employed. The pedestrian may move sequentially for several time steps and stop a similar long time, thus generating the oscillations depicted in Fig. 7. In addition, at the time when update probability is computed it cannot be known if the pedestrian will move to a side or a corner cell. Since the center-tocenter distance is different by $\sqrt{2}-1$, the desired velocity is not correctly reproduced in simulation. To im- 


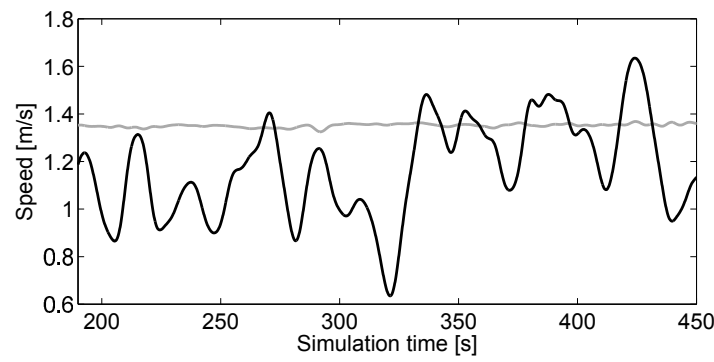

Fig. 7. Pedestrian velocity computed with (gray) and without (black) moving average correction (moving average has a period of $10 \mathrm{~s}$ ).

prove accuracy and precision of the walking speed we decided to introduce an additional algorithm, which is briefly presented in Fig. 8.

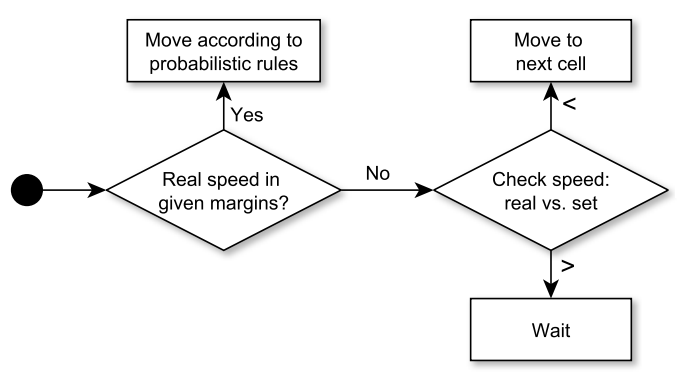

Fig. 8. Speed refinement algorithm for pedestrians.

At each time step the moving average of the real walking speed $v_{\text {avg }}$ is computed according to:

$$
v_{a v g}=\frac{d_{t o t} \cdot s_{c e l l}}{N_{a v g} \cdot t_{\text {step }}}
$$

where $d_{t o t}$ is total distance traveled in cell units (diagonal motion count as $\sqrt{2}$ ), $s_{\text {cell }}$ is the cell's side length, $N_{\text {avg }}$ the number of time steps considered and $t_{\text {step }}$ the time step length. To avoid cyclic oscillations, the size of $N_{\text {avg }}$ is slightly changed every time step. Once $v_{\text {avg }}$ has been computed, it is checked if the velocity lies within given margins of the desired walking speed. If this test is passed update is performed according to probabilistic rules (as shown above). If $v_{\text {avg }}$ is too large or too low, update is halted or forced accordingly to each case. As the gray line in Fig. 7 shows, the use of this algorithm allows to greatly stabilize the real walking speed.

To conclude the discussion about walking speed, it is important to remember that pedestrians do not only have different individual walking speeds, but also change their speed in accordance with each phase of the crossing attempt.

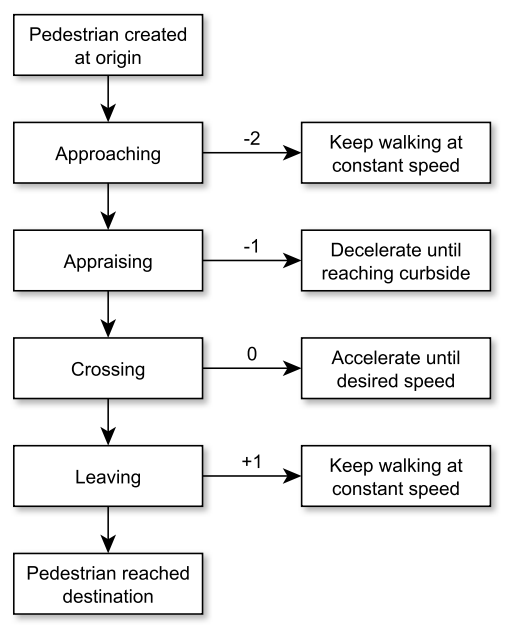

Fig. 9. Phase update and consequent actions (numbers indicate the phases used in the model).

In Fig. 9 the process used to update phase and change the desired speed is illustrated in detail. When a pedestrian is created it is decided if the given individual is an adult or an elderly according to the proportion of each group inside the population. Later, the desired walking speed is assigned to each pedestrian by using the distribution relative to the corresponding age group. By using the walking speed assigned, the pedestrian will move toward the crosswalk. At a given distance from the crosswalk (appraising distance), the pedestrian will start decelerating. The desired walking speed will consequently be changed every time step according to the given deceleration. When the pedestrian reaches the curbside, deceleration will stop and a decision will be taken on whether to cross or not. If safe conditions are met (details on the decision-making process are presented later) the pedestrian will accelerate until reaching its original walking speed. The destination is reached by keeping walk on that speed for the remaining time. For convenience, in the simulation model, a number is assigned to each phase as given in Fig. 9.

Finally, it has to be remarked that under certain conditions, it is possible that multiple pedestrians will attempt to move to the same cell. The algorithm used to avoid the overlapping of pedestrians is presented in Fig. 10. Keeping in mind the previous discussion, each time a position update is considered necessary, a pedestrian will use his floor field (which change 


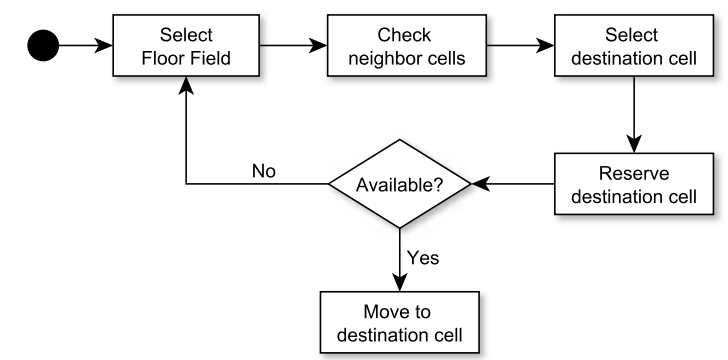

Fig. 10. Schematic representation of the pedestrian's moving algorithm.

depending on the destination) to select a destination within his neighborhood. The destination cell will be reserved first and after each pedestrian has selected his own destination it is checked if the given cell is exempt from conflicts. If the cell is available the pedestrian will move there. In case of conflicts one pedestrian will be picked up as winner among the candidates with equal probabilities. Only the winner will be allowed to move to the destination cells, the remaining candidates will have to pick up another cell.

\subsection{Vehicular model}

The car following computational method is based on a modified version of the Gipps model $[11,26]$ on which further changes have been made to allow the use of time steps different from the driver's reaction time. In the original formulation of the Gipps model it is assumed that drivers need a time step before reacting to changes occurring in the traffic. This constraint requires the use of a computational time step as close as possible to the typical driver's reaction time (usually in the order of $1 \mathrm{~s}$ ). When only cars are considered, this limitation does not cause any problem, since space is continuous (thus allowing accurate speed calculations) and on large vehicle fleets the use of constant reaction time is an acceptable approximation. However, since our model include pedestrians which move in a discrete space and need to change their velocity before and during the crossing attempts, choosing time steps equal to the reaction time would not be a satisfactory solution. For this reason, the Gipps model has been further modified allowing the use of time steps much smaller then the driver's reaction time.

According to the time-discrete Gipps model the maximum speed at which a car can travel without colliding with the front vehicle (or obstacle in general) is given by:

$$
v_{\text {safe }}=b\left(\alpha_{\text {safe }}+\beta_{\text {safe }}\right)
$$

where $b$ is the maximum breaking deceleration (given by the car's performances). $\alpha_{\text {safe }}$ and $\beta_{\text {safe }}$ are two functions defined as follows:

$$
\begin{aligned}
& \alpha_{\text {safe }}=\left[\sqrt{2 \frac{d_{p}+g}{b}+\frac{1}{4}}-\frac{1}{2}\right] \\
& \beta_{\text {safe }}=\frac{d_{p}+g}{\left(\alpha_{s a f e}+1\right) b}-\frac{\alpha_{\text {safe }}}{2}
\end{aligned}
$$

with $g$ being the distance with the front car and $d_{p}$ the minimum breaking distance which can be computed using:

$$
d_{p}=b\left(\alpha_{p} \beta_{p}+\frac{\alpha_{p}\left(\alpha_{p}-1\right)}{2}\right)
$$

where $\alpha_{p}$ and $\beta_{p}$ are defined as the integer and decimal part respectively of the ratio $v_{c a r} / b$, being the number of steps required to completely stop the car moving at speed $v_{c a r}$ (remember that $b$ is the maximum deceleration). As explained above, the reaction time is implicitly included in the fact that each driver can react to changes in the traffic conditions only at the next time step, thus requiring the time step being equal (or close) to the reaction time. To allow the use of time steps much smaller than the reaction time it is sufficient considering a gap $g_{n e w}$ equal to:

$$
g_{\text {new }}=g-t_{\text {reaction }} \cdot v_{\text {car }}
$$

in which the distance traveled by the car during the reaction time $t_{\text {reaction }} \cdot v_{\text {car }}$ is subtracted from the "real" gap from the front vehicle. By simply using the modified $g_{n e w}$ in Eqs. (4) and (5) this minor change allows to introduce different reaction times for each driver without having to heavily modify the Gipps model. The only condition for accurate results is to ensure that the time step used is sufficiently smaller than the smallest reaction time (this part will be discussed in the results).

The update of speed and position for each car is performed as indicated in the simple diagram of Fig. 11 


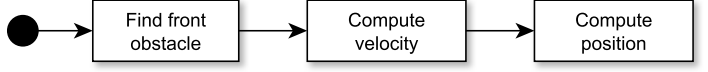

Fig. 11. Basic algorithm updating car position and speed.

and by using the update rules of the Gipps model (with some small modifications allowing to use different values for acceleration and breaking deceleration). For a given car with current speed $v_{c a r}(t)$, the velocity at the next time step can be obtained by initially compute $v_{1}$ and $v_{0}$ as follows:

$$
v_{1}=\min \left[v_{c a r}(t)+a, v_{\max }, v_{s a f e}\right]
$$

$$
v_{0}=v_{1}-\epsilon\left(v_{1}-\left(v_{c a r}(t)-a\right)\right)
$$

where $a$ is the maximum acceleration and $\epsilon$ is a model parameter typically chosen equal to 0.4 . The new velocity is finally computed by taking a random value between $v_{0}$ and $v_{1}$, or, in mathematical terms:

$$
v_{c a r}(t+1)= \begin{cases}v_{0}+\left(v_{1}-v_{0}\right) \cdot r, & \text { if } v_{0}<v_{1} \\ v_{1}+\left(v_{0}-v_{1}\right) \cdot r, & \text { otherwise }\end{cases}
$$

where $r$ is a randomly generated number between 0 and 1. As a last step it is necessary to check the speed change $v_{\text {car }}(t+1)-v_{\text {car }}(t)$ and, if required, set it to the maximum limits possible according to the car's performances. New position for each car is simply computed by multiplying the updated speed with the time step and adding the traveled distance to the old position.

\subsection{Car-pedestrian interaction}

Having discussed how pedestrians and cars move inside their own sub-environment, it is now important to present how both agents interact at the crosswalk, which is the main topic of this stud discussion by considering the decision making process for cars, which is summarized in Fig. 12.

At first, it is important to define if the considered vehicle is approaching the crosswalk or not. Obviously, vehicles which just passed the crosswalk do not need to pay attention on pedestrians attempting to cross it. If a queue of vehicles is approaching the crosswalk, it is assumed (supported by empirical evidence) that

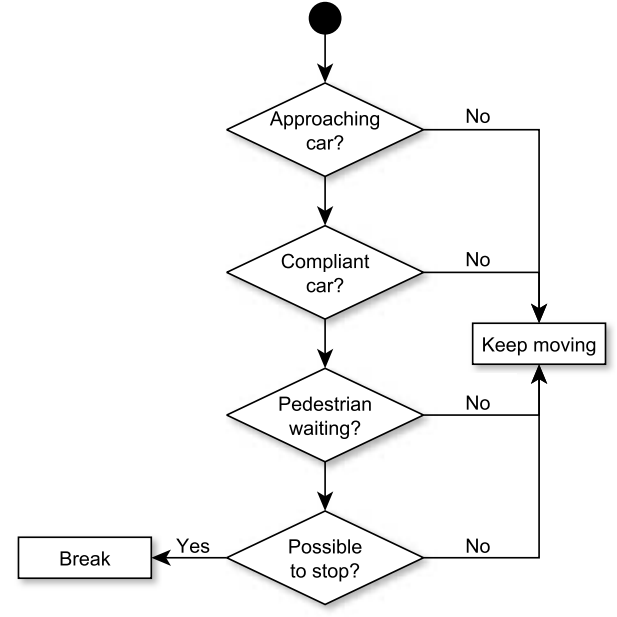

Fig. 12. Decision algorithm for cars' drivers.

only the leading vehicle will consider the presence of pedestrians. The remaining vehicles will only consider the front vehicle as an "obstacle" and adjust their speed accordingly. Next, we need to check if the approaching car is compliant or not. When vehicles are generated, compliance is assigned according to a given probability (obtained empirically). Non-compliant cars will ignore the presence of pedestrians at the curbside (or at the midblock) and will keep driving only caring of the distance from the front vehicle. Later, it has to be checked if a pedestrian is waiting on the relative side of the road. If not, there is no need to break before the crosswalk. Waiting pedestrians are only finally considered if all the previous conditions are met: approaching compliant car spotting pedestrian(s) waiting on the side of his lane. In this case, it is important to determine if the car is able to stop in the distance available before the crosswalk. This is performed by using the equations of the Gipps model. If the speed is too high to allow a complete stop, then the driver will abort his stopping attempt and instead adjust his speed by using the distance with the front vehicle. If the speed is low enough to allow a complete stop before the crosswalk, the driver will start breaking eventually arriving at a complete stop just before the crosswalk. As we will see later, pedestrians make similar considerations, which means that they will not attempt to cross if a vehicle cannot or do not want to stop.

Concerning pedestrians, the decision-making process for crossing is summarized in Fig. 13. After carefully examining the video recording it was concluded that a 2-step crossing mechanism would better sum- 


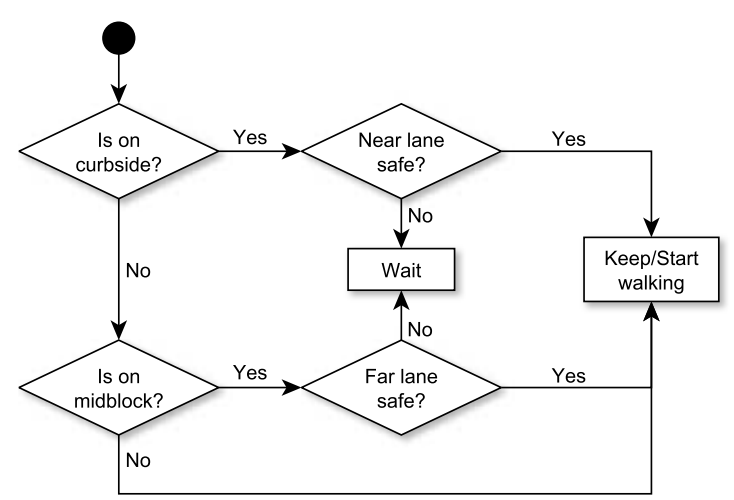

Fig. 13. Crossing decision algorithm for pedestrians near and on crosswalk.

marize the behavior observed in reality (a fact which was also supported by empirical evidence). Pedestrians studied will usually start crossing the road provided the near lane is safe. While crossing the road pedestrians will judge if stepping into the far lane is safe or not and will stop (or slow down) around the middle of the street if the incoming car is deemed as not breaking.

Therefore, the algorithm to assess if a pedestrian can start crossing or not has been designed as follows. At the beginning we need to check if a pedestrian is on the curbside, since crossing actions start from there. If so the pedestrian will check the safety of the near lane at first. For this check two different methods have been adopted as listed below.

- Method 1: calculation based on car speed and distance using the same equations of vehicles. If the speed is judged too high to stop in the space provided the crossing attempt is considered unsafe and the pedestrian will wait.

- Method 2: accepted time gap. When pedestrians are generated, together with the individual walking speed, a specific accepted time gap is assigned to each one according to the distributions for adults and elderly. Because of the statistical nature of distributions, a minimum value for accepted time gap is used to avoid insignificant values (negative numbers in particular). Before starting to cross, pedestrian compute the time gap by using speed and distance of the incoming vehicle. If the time gap is larger then the accepted one, then the pedestrian will step into the crosswalk.

Pedestrians judging the near lane as safe will start crossing and after entering the "virtual midblock" the decision-making process is repeated for the far lane.
When lanes are not judged as safe pedestrians will simply wait at the same position until safe conditions are met.

After discussing pedestrian, car and interaction modeling we can now consider how these aspects are combined in the computational loop which is presented in Fig. 14.

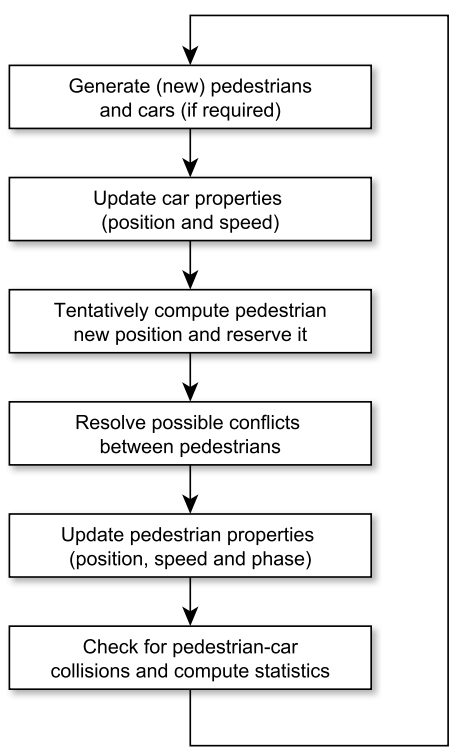

Fig. 14. Computational loop including update for pedestrians' and cars' properties.

Since vehicle and pedestrian sub-environment are independent it is possible to update each class of agent in a predetermined order. At first, if the number of pedestrians and/or cars is below the input given, pedestrians and/or cars are generated into each subenvironment. Next, each car's speed and position are updated in a sequential way by using the car-following model previously introduced. Here interactions with pedestrians will be considered while computing the speed of approaching vehicles. Later, pedestrians' new speed and position are also computed, but, since conflicts need to be solved first, pedestrians' do not move yet. Interaction with cars is considered to judge the next move of pedestrians on the curbside or on the midblock. The cell to move in is first reserved and conflicts resulting from pedestrians aiming at the same cells are resolved. Once cells reserved by each pedestrian do not overlap, pedestrians are moved in sequential way. Finally, it is check if collisions did not occur between pedestrians and vehicles on the crosswalk and output values are computed and stored. 


\subsection{Model parameters and constants}

To conclude the discussion about the simulation model, it is important to define the numerical values which has been used in simulations. This sections is divided into two parts: constants and parameter. Although, strictly speaking, both class of numbers can be changed for each simulation, constants are intended for those values which are typical for the scenario considered and are not intended for modification. For instance, walking speeds and driver reaction time are relative to human nature and will not change much (if not at all) if a different road with similar properties is chosen in a different area (these values will however change from country to country). On the other side, parameters refers here to conditions typical of the time of the day and the weather during which the observation was performed. For example, rainy weather will alter the breaking performance of cars and the flow of pedestrians will also change.

Model constants given in Table 5 were mostly obtained from the field study described above. Among the values taken from the literature is the reaction time $[25,33,45]$ and the pedestrian acceleration and deceleration [51]. Although values found in the literature are not specific for the scenario considered here, they refer to phenomena which only slightly change depending on the case considered. Model parameters are given in Table 6.

Some considerations need to be made here concerning the parameters used. During the observation only car flow has been measured. However, by assuming that traffic conditions allowed to drive at a maximum speed of $35 \mathrm{~km} / \mathrm{h}$ (data taken from video recording), the relative density can be computed. Car performances are typical of mid-range vehicles. Pedestrian flow refers to the frequency of crossing attempts per minute. Since our simulation model do not consider group cohesion, multiple pedestrians crossing in group in the field study are considered as a single entity (see also previous discussion). Finally, the simulated time for one simulation run has been chosen equal to the total length of the observation.

\section{Model validation and results}

To check out the capabilities of the model developed and in particular assess its accuracy in estimating motion of car and pedestrians, different cases have been considered. First of all, it is important to check if the
Table 5

Constants used in the model

\begin{tabular}{|c|c|}
\hline \multicolumn{2}{|l|}{ General constants } \\
\hline Time step & $0.1 \mathrm{~s}$ \\
\hline Pedestrian cell size & $0.4 \mathrm{~m}$ \\
\hline \multicolumn{2}{|l|}{ Scenario dimensions } \\
\hline Road length & $374 \mathrm{~m}$ \\
\hline Sidewalk width & $2.4 \mathrm{~m}$ \\
\hline Lane width & $4.8 \mathrm{~m}$ \\
\hline Crosswalk width & $3.6 \mathrm{~m}$ \\
\hline Mid-lane width & $1.2 \mathrm{~m}$ \\
\hline \multicolumn{2}{|l|}{ Car dynamic } \\
\hline Car length & $4.5 \mathrm{~m}$ \\
\hline Minimum gap & $1.0 \mathrm{~m}$ \\
\hline Driver reaction time (mean) & $1.1 \mathrm{~s}$ \\
\hline Driver reaction time (variance) & $0.2 \mathrm{~s}$ \\
\hline \multicolumn{2}{|l|}{ Pedestrian dynamic } \\
\hline Adult walking speed (mean) & $1.30 \mathrm{~m} / \mathrm{s}$ \\
\hline Adult walking speed (variance) & $0.20 \mathrm{~m} / \mathrm{s}$ \\
\hline Elderly walking speed (mean) & $1.05 \mathrm{~m} / \mathrm{s}$ \\
\hline Elderly walking speed (variance) & $0.20 \mathrm{~m} / \mathrm{s}$ \\
\hline Acceleration & $0.30 \mathrm{~m} / \mathrm{s}^{2}$ \\
\hline Deceleration & $0.50 \mathrm{~m} / \mathrm{s}^{2}$ \\
\hline \multicolumn{2}{|l|}{ Crossing behavior } \\
\hline Appraising distance & $3.0 \mathrm{~m}$ \\
\hline Accepted gap adult (mean) & $4.0 \mathrm{~s}$ \\
\hline Accepted gap adult (variance) & $2.5 \mathrm{~s}$ \\
\hline Accepted gap elderly (mean) & $4.5 \mathrm{~s}$ \\
\hline Accepted gap elderly (variance) & $1.8 \mathrm{~s}$ \\
\hline Minimum accepted gap & $1.0 \mathrm{~s}$ \\
\hline
\end{tabular}

modifications made on the Gipps model allow to correctly estimate the dynamics found in vehicular traffic.

To qualitatively assess the degree of congestion in a given road, the fundamental diagram is typically employed. Average speed and flow are measured during a defined time period (usually 5 or 10 minutes) in the section of the road which is to be analyzed. Density can also be obtained experimentally but it is usually computed by dividing the flow with the average speed. The result for a single measurement of flow and density is plotted in a diagram, resulting in a dispersion of points like the one shown in Fig. 15. In Fig. 15 the result for a measurement in an highway and a local road are plotted. Both cases show a fairly linear relationship with density and flow, translating into a smooth traffic flow. Since speed limits are different, the slope of each diagram is also different: $100 \mathrm{~km} / \mathrm{h}$ for the highway and $50 \mathrm{~km} / \mathrm{h}$ for the local road. 
Table 6

Parameters used in the model

\begin{tabular}{ll}
\hline \multicolumn{2}{c}{ Car related } \\
\hline Car density & $16.30 \mathrm{cars} / \mathrm{km} / \mathrm{lane}$ \\
Maximum (breaking) deceleration & $9.0 \mathrm{~m} / \mathrm{s}^{2}$ \\
Maximum acceleration & $2.0 \mathrm{~m} / \mathrm{s}^{2}$ \\
Maximum (possible) speed & $35 \mathrm{~km} / \mathrm{h}$ \\
Non-compliant drivers ratio & $0.5(50 \%)$ \\
\hline \multicolumn{2}{c}{ Pedestrian related } \\
\hline Pedestrian flow (both directions) & $5.52 \mathrm{~min}{ }^{-1}$ \\
Ratio of elderly pedestrians & $0.25(25 \%)$ \\
\hline \multicolumn{2}{c}{ Other parameters } \\
\hline Simulated time (one run) & $4500 \mathrm{~s}(1 \mathrm{~h}: 15 \mathrm{~m})$ \\
\hline
\end{tabular}

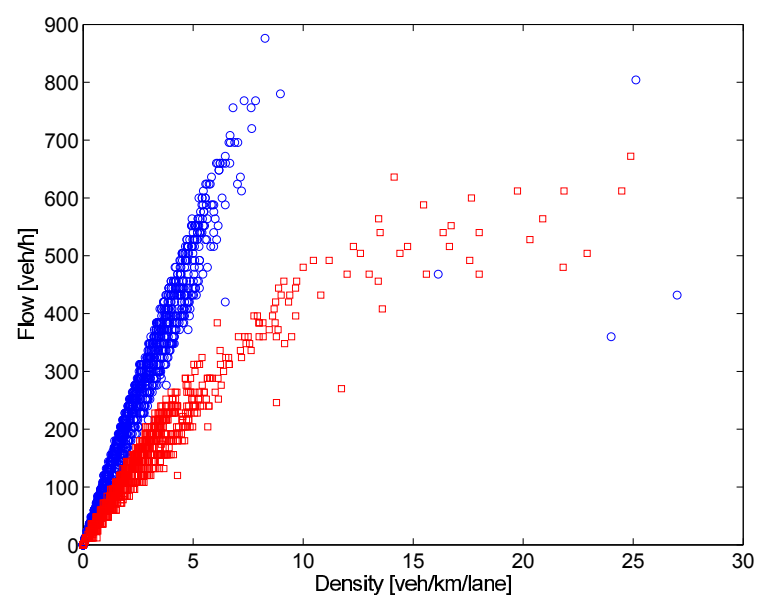

Fig. 15. A traffic fundamental diagram for an highway (circles) and a urban road (dots) during free (or low congested) flow [2].

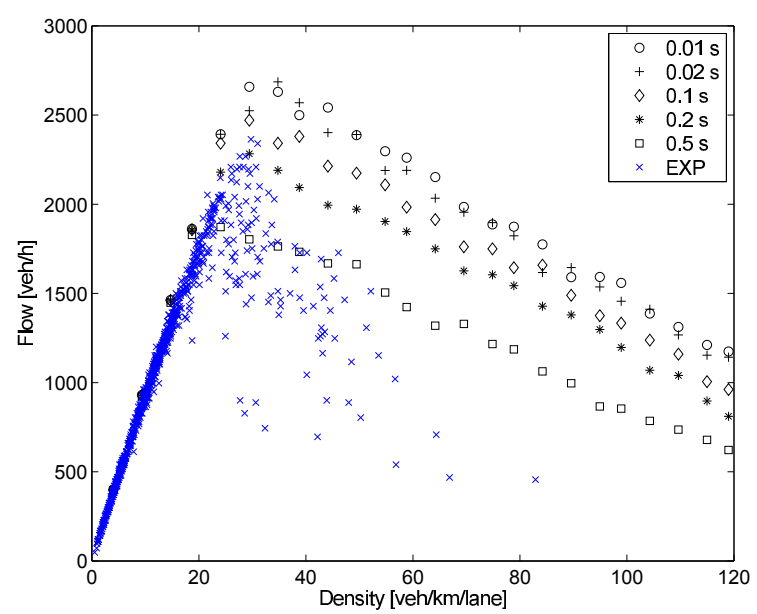

Fig. 16. Simulated car fundamental diagram for an highway with 100 $\mathrm{km} / \mathrm{h}$ speed limit (density vs. flow). Blue crosses are experimental data.

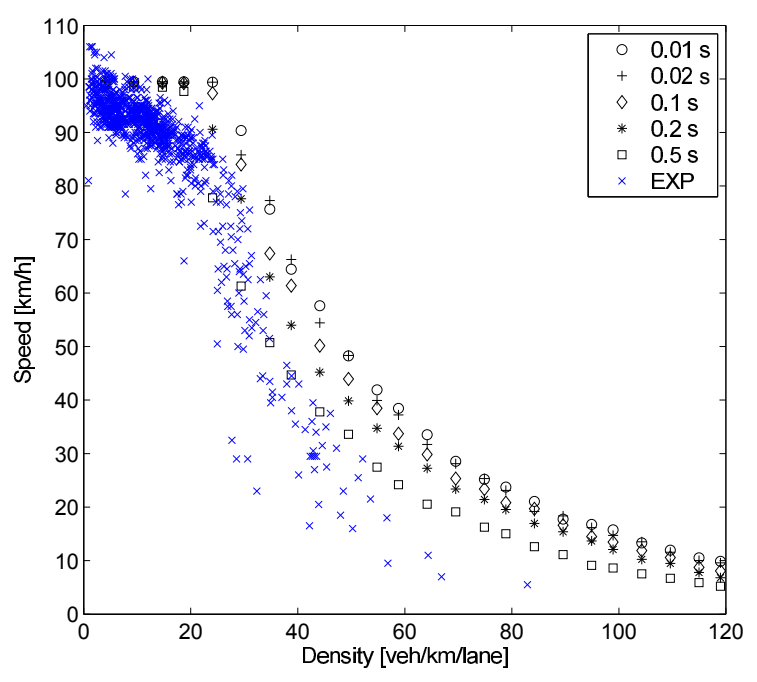

Fig. 17. Simulated car fundamental diagram for an highway with 100 $\mathrm{km} / \mathrm{h}$ speed limit (density vs. speed). Blue crosses are experimental data.

To validate the vehicular model used in this study, simulations without pedestrians were performed by changing car density and time step. Results for the highway case with a speed limit of $100 \mathrm{~km} / \mathrm{h}$ are plotted in Fig. 16 and Fig. 17, showing the density-flow and density-speed diagram respectively. Results were compared with experimental data taken in a section of highway where traffic jams routinely occur. It can be noticed that the fundamental diagram does not significantly change when small time steps are used, meaning that the modifications applied on the Gipps model allow to use variable time steps. However, when the time step grows close to the reaction time a significant reduction in the maximum flow is observed, which is to be expected since the remainder of the division between reaction time and time steps becomes large.

It is remarkable to notice the accuracy in predicting the transition between free-flow and congestion, which is observed in Fig. 16 when the initially linearly growing flow reach a maximum and capacity drops. Both experimental and simulated data agree with a transition around 30 vehicles $/ \mathrm{km}$. The maximum flow reached is predicted with less accuracy, but a time step of 0.1 or 0.2 seconds accurately reproduce the experimental case. The simulation model partially fails in computing the behavior of congested flow, but if results are analyzed in term of speed and density (as shown in Fig. 17), it can be concluded that the accuracy is sufficient (especially considering that mostly uncongested flow is observed near the crosswalk). 


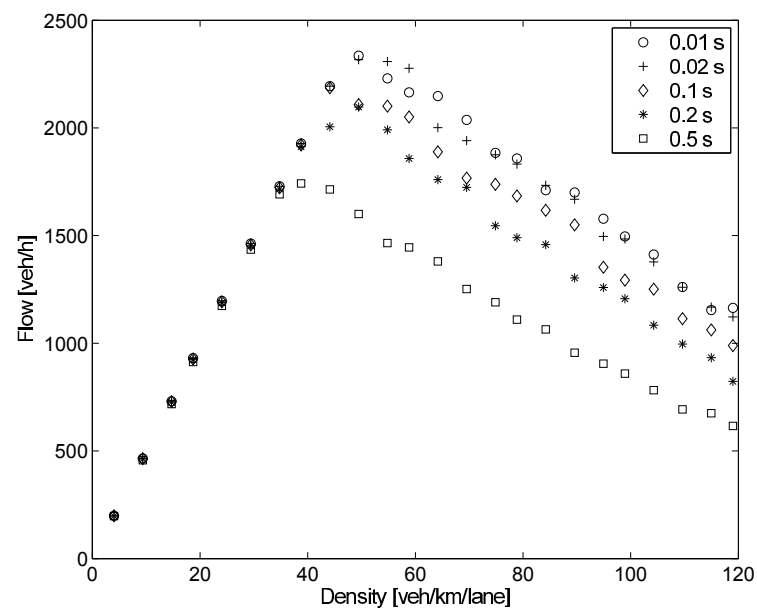

Fig. 18. Simulated car fundamental diagram for an urban road with $50 \mathrm{~km} / \mathrm{h}$ speed limit (density vs. flow).

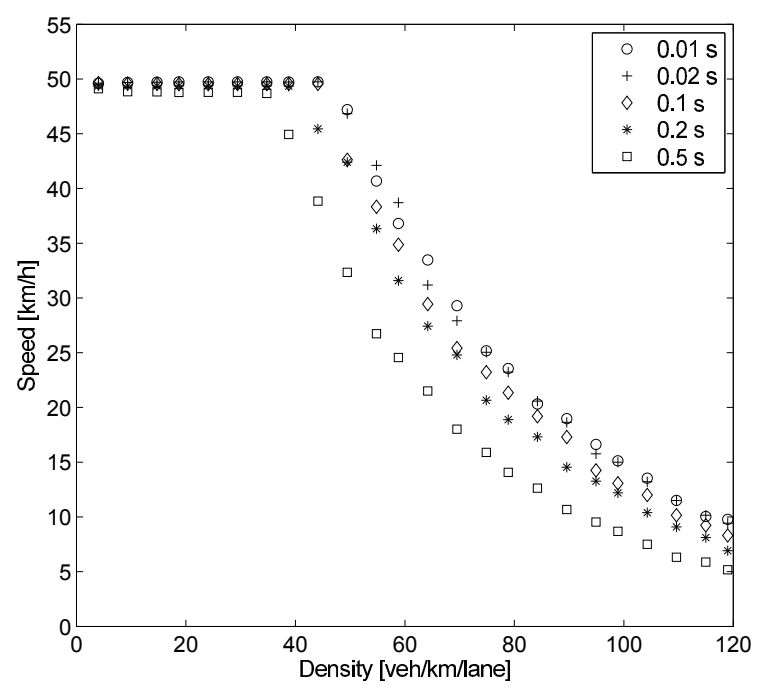

Fig. 19. Simulated car fundamental diagram for an urban road with $50 \mathrm{~km} / \mathrm{h}$ speed limit (density vs. speed).

In Fig. 18 and Fig. 19 the results for simulated fundamental diagrams are presented for the case of an urban road (with $50 \mathrm{~km} / \mathrm{h}$ speed limit), which is more similar to the case considered in this study, but for which large experimental data are not available. Nonetheless, results of the $50 \mathrm{~km} / \mathrm{h}$ case seem to agree with some simple values from the literature [35], with transition density lying around 40 vehicles $/ \mathrm{km}$ and a maximum flow of slightly more than 2000 vehicles/hour/lane. Again, in the $50 \mathrm{~km} / \mathrm{h}$ case also, results are similar when the time step is chosen in the $0.01-0.2$ $\mathrm{s}$ interval and become significantly different for rela- tively large time steps (as the case of $0.5 \mathrm{~s}$ ).

Having verified that the vehicular model allows to correctly simulate car traffic behavior it is now important to check if the pedestrian model allows to include the features for which has been developed. In particular, since modeling elderly pedestrian is part of the motivation of this study, we want to check if pedestrians with different velocities are correctly reproduced in the discrete computational space. As discussed before, some specific algorithms were required to assure that pedestrians effectively move at the desired speed and, since distributions are employed, we want to verify if a population of pedestrians actually move with different walking speeds.

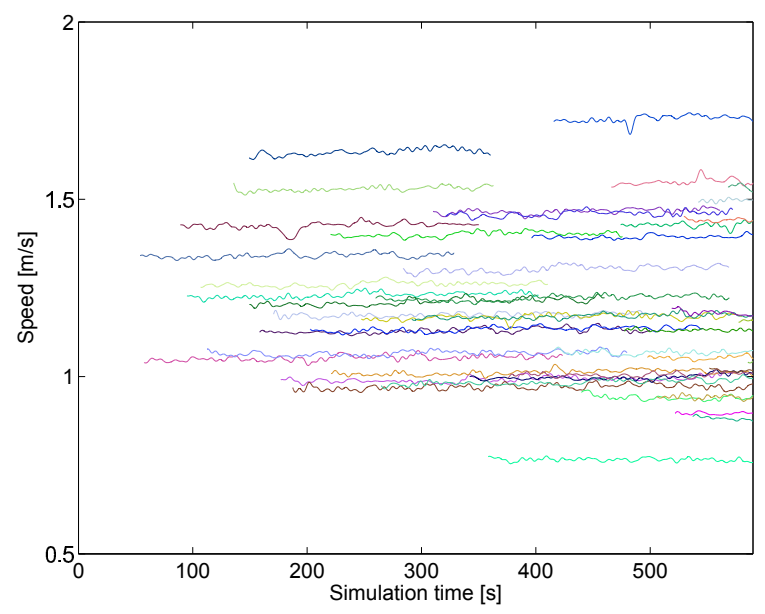

Fig. 20. Pedestrians' speed during a simulation with different age groups (velocities are averaged over a $10 \mathrm{~s}$ interval, pedestrians characteristics are given in Table 5).

In Fig. 20 walking speeds for different pedestrians are plotted against simulation time. Each line corresponds to a single pedestrian which entered the simulation scenario when the line started and was able to reach the "exit" at the end of the line. Two considerations can be done by observing Fig. 20. First of all, it can be concluded that the algorithm previously presented allows to stabilize pedestrians speed, creating a stable trend similar to situations observed in reality. Next, it can be observed that it is possible to differentiate between individual pedestrian velocities, meaning that it is possible to consider differences between adults and elderly when passing through the crosswalk. However, because of the relatively long moving average interval used, velocity fluctuations during the different crossing phases could not be observed. To observe this type of variations, small time intervals would 
have to be used when computing the moving average, but this would result in fluctuations which cannot be removed because of the discrete modeling approach. A solution to see small term velocity fluctuations would be to use a very small mesh size, but computational time dramatically increases and unrealistic pedestrian densities may form during the simulation.

Finally, we would like to check the validity of the modeling approach used to simulate vehicle-pedestrians interactions. As a comparative quantity to analyze differences between the simulation model and the behavior observed in reality, the delay (or time loss) used to evaluate the Level of Service can be used. Results for vehicular and pedestrian delay and flow of both observation and simulation are presented in Table 7 .

A set of 100 simulation runs were performed for both methods previously discussed on the pedestrian decision-making process. Method 1 (comparison between vehicle speed and maximum speed allowed for a complete stop) clearly performs better than method 2 (accepted time gap), with delays computed with method 1 lying within the margin of observed quantities. Pedestrian flow appears to be consistent with both methods, but, being an input parameter not related with vehicle interaction is not representative of the quality of the model and it is only reported for reference purpose. Simulated vehicular flow (which strongly depends on the interaction with crossing pedestrians) is lower than the empirical figure, but it is important to remark that observed flow include a variety of vehicles (cars, bikes, cycles,...), while the model has been developed on the assumption that only cars are moving on the road. For example, a bicycle can safely pass on the crosswalk when a pedestrian is walking, a behavior not included in the simulation model. If only cars (or 4-wheel vehicles) are accounted for, then the resulting figure for the observed flow will be lowered, getting closer to the simulated value.

The reason for the high delays computed with method 2 may be related to the fact that the distribution of time gaps resulting from the observation is not correctly reproduced in the simulation, where a normal distribution is used. Although, the concept of time gap is particularly useful in measuring for example differences between adult and elderly, a direct use in simulation may not be appropriate. Possibly, an extension of the concept of time gap by using different distributions for the accepted and refused time gap as proposed by some researchers [24] may help improving the accuracy of the results. The good agreement between observed delay and the one computed with method 1 possibly sug- gest that, as some researcher found [44], under clear weather conditions and for low vehicles speeds (like the case of the observation) pedestrian can correctly estimate breaking distance of cars.

Nonetheless, although numerical values for method 2 are much higher than observed delays, results are closest to reality when the Level of Service is adopted. Delays measured in the field study are very low, corresponding to a level A for both vehicular and pedestrian traffic. Both method 1 and 2 are able to obtain the same result numerically (level A). For the vehicular traffic method 2 is only one level above the one observed, meaning that from a qualitative point of view this result can still be considered as satisfactory. In other words, we can conclude that the model developed allowed to correctly assess the LOS found in unsignalized crosswalks (especially when method 1 is employed), thus making it feasible to employ it to assess, for example, if in a given location an unsignalized crosswalk would perform better than a traffic light in term of quality of service perceived by road users. The particularity of considering elderly pedestrian in the simulation would allow to assess with a better accuracy different area of a city characterized with different types of age groups.

\section{Conclusions and discussion}

Based on empirical results from a field survey, a simulation model for pedestrian crossing at unsignalized crosswalks was developed. Although both vehicular and pedestrian motion is based on classical models found in the literature, specific modifications were required to allow considering important aspects related to vehicle-pedestrian interaction. Concerning pedestrians, small time steps were required to allow considering different walking speeds and therefore making it possible to differentiate between different age group (adult and elderly in particular). In addition, a refinement of the update algorithm was required to remove large fluctuations found in the motion of pedestrians inside the discrete computational grid. Concerning cars, the variable time steps used for pedestrians resulted in the requirement to modify the Gipps carfollowing model. Driver's reaction time has been explicitly included in the computational loop, also making it possible to consider a distribution for the reaction time which closely reproduce drivers' behavior. Crossing mechanism of pedestrians has been modeled in a two-step fashion to reproduce behavior observed in the field study. A pedestrian attempting to cross will 
Table 7

Simulation results for vehicle-pedestrian interaction at crosswalk (values used in the simulation are given in Table 5 and Table 6), letters in brackets refer to the Level of Service

\begin{tabular}{llll}
\hline \multirow{2}{*}{ Quantity observed } & \multirow{2}{*}{ Observation } & \multicolumn{2}{c}{ Simulation } \\
& & \multicolumn{1}{c}{ Method 1 } & \multicolumn{1}{c}{ Method 2 } \\
\hline Vehicle delay & $3.20 \pm 2.73 \mathrm{~s} \mathrm{(A)}$ & $3.96 \pm 0.43 \mathrm{~s}(\mathrm{~A})$ & $6.81 \pm 1.29 \mathrm{~s}(\mathrm{~B})$ \\
Pedestrian delay & $1.29 \pm 0.21 \mathrm{~s} \mathrm{(A)}$ & $1.28 \pm 0.26 \mathrm{~s}(\mathrm{~A})$ & $5.86 \pm 0.99 \mathrm{~s}(\mathrm{~A})$ \\
\hline Vehicle flow & 1139 & $1013 \pm 11 \mathrm{veh} / \mathrm{h}$ & $950 \pm 29 \mathrm{veh} / \mathrm{h}$ \\
Pedestrian flow & 5.52 & $5.52 \pm 0.12 \mathrm{~min}^{-1}$ & $5.53 \pm 0.11 \mathrm{~min}^{-1}$ \\
\hline
\end{tabular}

consider only the near lane first and later check the safety of the far one while walking on the crosswalk. Results from simulations show that a good agreement with delay of both road users is found when pedestrians are supposed to estimate velocity of incoming vehicles and judge if they can stop in the given distance. The use of the accepted gap also led to somehow acceptable results, but the concept need to be investigated further to allow a successful implementation in simulation.

To conclude the discussion, it is important to remark that the model developed in the frame of this research is representative of the phenomena observed during the field study in Milan. This means that, although generally speaking behavior of pedestrians will not largely change in different countries, the accuracy of the model may be limited if, for example, high noncompliance rates are found in drivers or the traffic is composed by a large number of 2-wheels vehicles. Nonetheless, the approach presented here will surely help developing a partially different model representing the specific behavior found in another country/city. A possible application of the unsignalized crosswalk model may be in city planning. In particular, this model can be useful to determine where to place a crosswalk and which LOS can be expected in the given location. Most of the constants used in the simulation will not change much, especially inside the same city, and expected pedestrian and vehicular flow can be estimated by means of measurements or surveys. With both flows provided, the simulation can easily allow to predict the LOS and thus help decision-makers determining if an unsignalized crosswalk is appropriate or alternative solutions have to be considered (underground passage, traffic light,...). The model may also be used to assess safety of pedestrians, especially where large populations of elderly are found.

In the future this model could be further improved by introducing mechanisms reproducing collisions, which are excluded at the moment. Although this implemen- tation will further increase the application range, experimental data are required to calibrate the model. This may be the most difficult part, since empirical data for pedestrian-car collisions are only available at the macroscopic scale.

\section{Acknowledgments}

This research has been mostly founded by the Italian Association for Artificial Intelligence $(A I * I A)$ with the “AI*IA INCOMING MOBILITY GRANTS 2016". In addition, the Foundation for Supporting International Students and the Doctoral Student Special Incentives Program (SEUT RA) of the University of Tokyo have also financially contributed to part of this research.

\section{References}

[1] A. S. Al-Ghamdi. Pedestrian-vehicle crashes and analytical techniques for stratified contingency tables. Accident Analysis \& Prevention, 34(2):205-214, 2002.

[2] M. I. Ali, F. Gao, and A. Mileo. Citybench: a configurable benchmark to evaluate rsp engines using smart city datasets. In International Semantic Web Conference, pages 374-389. Springer, 2015.

[3] L. Asher, M. Aresu, E. Falaschetti, and J. Mindell. Most older pedestrians are unable to cross the road in time: a crosssectional study. Age and ageing, 41(5):690-694, 2012.

[4] S. Bandini, L. Crociani, and G. Vizzari. Pedestrian simulation: Considering elderlies in the models and in the simulation results. In Ambient Assisted Living, pages 11-21. Springer, 2015.

[5] L. Crociani and G. Vizzari. An integrated model for the simulation of pedestrian crossings. In Cellular Automata: 11th International Conference on Cellular Automata for Research and Industry, ACRI 2014, Krakow, Poland, September 22-25, 2014. Proceedings, pages 670-679. Springer International Publishing, 2014.

[6] C. F. Daganzo and V. L. Knoop. Traffic flow on pedestrianized streets. Transportation Research Part B: Methodological, $86: 211-222,2016$

[7] Department for Transport. Road Casualties Great Britain: 2006 - Annual Report, 2007. 
[8] Department for Transport. Reported Road Casualties Great Britain: 2014 - Annual Report, 2015.

[9] D. Evans and P. Norman. Understanding pedestrians' road crossing decisions: an application of the theory of planned behaviour. Health Education Research, 13(4):481-489, 1998.

[10] C. Feliciani and K. Nishinari. Empirical analysis of the lane formation process in bidirectional pedestrian flow. Phys. Rev. E, 94:032304, 2016

[11] P. G. Gipps. A behavioural car-following model for computer simulation. Transportation Research Part B: Methodological, 15(2):105-111, 1981.

[12] B. H. Goh, K. Subramaniam, and Y. T. Wai. Pedestrian crossing speed: the case of malaysia. In International Journal for Traffic and Transport Engineering, volume 2. Scientific Research Center Ltd., 2012.

[13] S. V. Gomes. The influence of the infrastructure characteristics in urban road accidents occurrence. Accident Analysis \& Prevention, 60:289-297, 2013.

[14] A. Gorrini, G. Vizzari, and S. Bandini. Towards modelling pedestrian-vehicle interactions: Empirical study on urban unsignalized intersection. arXiv preprint arXiv:1610.07892, 2016.

[15] J. D. Griffiths. A mathematical model of a nonsignalized pedestrian crossing. Transportation Science, 15(3):222-232, 1981.

[16] M. M. Hamed. Analysis of pedestrians' behavior at pedestrian crossings. Safety Science, 38(1):63-82, 2001.

[17] J. Hatfield and S. Murphy. The effects of mobile phone use on pedestrian crossing behaviour at signalised and unsignalised intersections. Accident Analysis \& Prevention, 39(1):197-205, 2007.

[18] D. Helbing, R. Jiang, and M. Treiber. Analytical investigation of oscillations in intersecting flows of pedestrian and vehicle traffic. Physical Review E, 72(4):046130, 2005.

[19] L. Henderson and D. Lyons. Sexual differences in human crowd motion. Nature, 1972.

[20] N. I. Ibrahim, F. A. Kidwai, and M. R. Karim. Motorists and pedestrian interaction at unsignalised pedestrian crossing. In Proceedings of the Eastern Asia society for transportation studies, volume 5, pages 120-125, 2005.

[21] Istituto nazionale di statistica. Incidenti stradali in Italia nel 2015, 2016.

[22] Istituto nazionale di statistica \& Automobile Club d'Italia. Le statistiche degli incidenti stradali in Italia dagli anni trenta ad oggi, 2011.

[23] E. Jarawan, D. Mohan, A. A. Hyder, M. Peden, R. Scurfiled, and D. Sleet. World report on road traffic injury prevention, 2004.

[24] S. Jiangang, W. Zhenhua, and C. Yanyan. Analysis on behaviors and safety of vrus at unsignalized roadway crosswalk. In ICTCT Extra Workshop, Beijing, 2008

[25] R. S. Jurecki, M. Jaśkiewicz, M. Guzek, Z. Lozia, and P. Zdanowicz. Driver's reaction time under emergency braking a car-research in a driving simulator. Eksploatacja $i$ Niezawodność, 14:295-301, 2012.

[26] S. Krauss, P. Wagner, and C. Gawron. Metastable states in a microscopic model of traffic flow. Physical Review E, 55(5):5597, 1997.

[27] A. T. Lawniczak, B. N. Di Stefano, and J. B. Ernst. Stochastic model of cognitive agents learning to cross a highway. In Stochastic Models, Statistics and Their Applications, pages
319-326. Springer, 2015.

[28] A. T. Lawniczak, J. B. Ernst, and B. N. Di Stefano. Creature learning to cross a ca simulated road. In Cellular Automata, pages 425-433. Springer, 2012

[29] R. Lobjois, N. Benguigui, and V. Cavallo. The effects of age and traffic density on street-crossing behavior. Accident Analysis \& Prevention, 53:166-175, 2013.

[30] R. Lobjois and V. Cavallo. Age-related differences in streetcrossing decisions: The effects of vehicle speed and time constraints on gap selection in an estimation task. Accident Analysis \& Prevention, 39(5):934-943, 2007.

[31] H. C. Manual et al. Transportation research board, 2000.

[32] Marisamynathan and V. Perumal. Study on pedestrian crossing behavior at signalized intersections. Journal of Traffic and Transportation Engineering (English Edition), 1(2):103-110, 2014.

[33] A. Mehmood and S. M. Easa. Modeling reaction time in car-following behaviour based on human factors. International Journal of Applied Science, Engineering and Technology, 5(14):93-101, 2009.

[34] K. Nagel, P. Wagner, and R. Woesler. Still flowing: Approaches to traffic flow and traffic jam modeling. Operations research, 51(5):681-710, 2003.

[35] K. Nagel, D. E. Wolf, P. Wagner, and P. Simon. Two-lane traffic rules for cellular automata: A systematic approach. Physical Review E, 58(2):1425, 1998.

[36] E. Papadimitriou, G. Yannis, and J. Golias. A critical assessment of pedestrian behaviour models. Transportation research part $F$ : traffic psychology and behaviour, 12(3):242255, 2009.

[37] H. J. Park, S. Li, W. Yu, W. Yang, W. Alhajyaseen, and M. IryoAsano. Pedestrian crossing behavior and compliance at signalized intersections. In 17th International Conference Road Safety On Five Continents (RS5C 2016), Rio de Janeiro, Brazil, 17-19 May 2016. Statens väg-och transportforskningsinstitut, 2016.

[38] C. G. Prato, V. Gitelman, and S. Bekhor. Mapping patterns of pedestrian fatal accidents in israel. Accident Analysis \& Prevention, 44(1):56-62, 2012.

[39] D. L. Roenker, G. M. Cissell, K. K. Ball, V. G. Wadley, and J. D. Edwards. Speed-of-processing and driving simulator training result in improved driving performance. Human Factors: The Journal of the Human Factors and Ergonomics Society, 45(2):218-233, 2003.

[40] I. Santé, A. M. García, D. Miranda, and R. Crecente. Cellular automata models for the simulation of real-world urban processes: A review and analysis. Landscape and Urban Planning, 96(2):108-122, 2010

[41] J. R. Short and L. M. Pinet-Peralta. No accident: Traffic and pedestrians in the modern city. Mobilities, 5(1):41-59, 2010.

[42] V. P. Sisiopiku and D. Akin. Pedestrian behaviors at and perceptions towards various pedestrian facilities: an examination based on observation and survey data. Transportation Research Part F: Traffic Psychology and Behaviour, 6(4):249274, 2003.

[43] M. Sporchia. Simulazione integrata dell'interazione tra pedoni e veicoli in intersezioni regolate e non. Master's thesis, Università degli Studi di Milano Bicocca, 2013.

[44] R. Sun, X. Zhuang, C. Wu, G. Zhao, and K. Zhang. The estimation of vehicle speed and stopping distance by pedestrians crossing streets in a naturalistic traffic environment. Trans- 
portation research part $F$ : traffic psychology and behaviour, 30:97-106, 2015.

[45] G. T. Taoka. Brake reaction times of unalerted drivers. ITE journal, 59(3):19-21, 1989.

[46] U.S. Department of Transportation. Traffic Safety Facts - 2013 Data. U.S. Department of Transportation, 2015.

[47] A. Várhelyi. Drivers' speed behaviour at a zebra crossing: a case study. Accident Analysis \& Prevention, 30(6):731-743, 1998.

[48] J. Wąs, J. Porzycki, R. Lubaś, J. Miller, and G. Bazior. Agentbased approach and cellular automata - a promising perspective in crowd dynamics modeling? In Acta Physica Polonica B Proceedings Supplement, volume 9. Jagiellonian University, 2016.
[49] U. Weidmann. Transporttechnik der Fussgänger: Transporttechnische Eigenschaften des Fussgängerverkehrs (Literaturauswertung). ETH, IVT, 1993.

[50] J. Yang, W. Deng, J. Wang, Q. Li, and Z. Wang. Modeling pedestrians' road crossing behavior in traffic system microsimulation in china. Transportation Research Part A: Policy and Practice, 40(3):280-290, 2006.

[51] J. Zębala, P. Ciępka, and A. Reza. Pedestrian acceleration and speeds. Problems of Forensic Sciences, 91:227-234, 2012.

[52] W. Zeng, P. Chen, H. Nakamura, and M. Iryo-Asano. Application of social force model to pedestrian behavior analysis at signalized crosswalk. Transportation research part $C$ : emerging technologies, 40:143-159, 2014. 